Research Article

\title{
Reliability Evaluation of Multiple DCFP System subject to Shifting Threshold
}

\author{
Chunping Li, ${ }^{1}$ Huibing Hao $\left(D,{ }^{1}\right.$ Fang $\mathrm{Xu}^{2}{ }^{2}$ and Guotao $\mathrm{Zhao}^{2}$ \\ ${ }^{1}$ School of Mathematics and Statistics, Hubei Engineering University, Xiaogan, Hubei, China \\ ${ }^{2}$ School of Computer and Information Science, Hubei Engineering University, Xiaogan, Hubei, China \\ Correspondence should be addressed to Huibing Hao; haohuibing1979@126.com
}

Received 6 February 2020; Revised 16 June 2020; Accepted 25 June 2020; Published 14 July 2020

Academic Editor: Francesco Franco

Copyright (c) 2020 Chunping Li et al. This is an open access article distributed under the Creative Commons Attribution License, which permits unrestricted use, distribution, and reproduction in any medium, provided the original work is properly cited.

This paper focuses on system reliability analysis with dependent competing failure process due to soft failure and hard failure. Some new probabilistic methods based on cumulative shock model and nonlinear Wiener process under different shifting thresholds situation are obtained. Considering that nonlinearity exists extensively in practice, the continuous soft failure process is governed by random effected nonlinear Wiener process. Firstly, reliability evaluation models for hard failure and soft failure are obtained under the cumulative shock, respectively. Furthermore, some system reliability models under different shifting thresholds situation are studied, in which failure threshold will decrease after a certain number of shocks. A real numerical example about fatigue crack growth dataset is carried out to demonstrate the proposed procedure. Numerical results indicate that both random shocks and shifting threshold have significant effect on system reliability. Finally, some sensitivity analysis are also been given.

\section{Introduction}

In actual operation environment, most of products are often exposed to different types of risk. Those risks may come from products themselves or external environments, and the same risk can also result in different failure categories. Specifically, we consider two failure processes: soft failure process and hard failure process, where the soft failure is caused by a gradual degradation and additional abrupt degradation damages due to a shock process, and hard failure is caused by an abrupt stress from the same shock process. As we know that these two failure processes exist simultaneously for most products (or systems). Therefore, the failure of product is usually the result of the competition between them, and we call it competing failure.

In recent years, theoretical studies for competing failure have been extensively explored in reliability analysis. Li and Pham [1] conducted system reliability analysis subject to degradation and shock process. Lehman [2] studied the competing failure model due to degradation and random shock. Klutke and Yang [3] studied the availability of maintained systems affected by the degradation process and random shock process. Zhu et al. [4] established an independent competing failure model based on sudden failure and degradation process. In [5], a continuous degradation system was proposed by considering soft failure and hard failure. In other studies, several random shock models are constructed, such as cumulative shock model $[6,7]$, extreme shock model [8], mixed model [9], and $\delta$-shock model [10, 11]. Most previous studies assumed that the two failure processes were independent. However, in actual operation circumstances, these two failure processes may be dependent because the effects from the same shock process contribute to both failure processes. When they are dependent, it creates a new problem to analyze the system reliability.

Recently, the reliability analysis of system with multiple dependent competing failure processes (DCFP) has been analyzed by many researchers. Peng et al. [12] introduced reliability modeling for systems with multiple DCFP. Wang et al. [13] proposed the dependent reliability assessment model under the degradation and shock process. Qiu and Cui [14] proposed the dependent two-stage failure process model with competing failures. Song et al. [15] considered multicomponent DCFP. Raflee et al. [16] proposed a reliability 
model for devices subject to DCFP with the changing degradation rate. An and Sun [17] proposed the reliability model for system experiencing DCFP with shock loads above a certain level. Hao and Yang [18] proposed a reliability analysis for DCFP with changing degradation rate and hard failure threshold levels. Fan et al. [19] proposed Bayesian approach for remaining useful life prediction of DCFP. Kong et al. [20] developed reliability models with sensor-based calibrations with multiple DCFP. Gao et al. [21] considered reliability models subject to DCFP with degradation-shock dependences. In all of these DCFP models, the failure thresholds are considered to be fixed constants, implying that the component resistance to failure does not deteriorate as the system ages. However, when the system sustains a series of shocks, the product may become more vulnerable and its resistance to failure is weakening. Therefore, the failure threshold decreases after multiple shocks, but only few papers considered the changing failure threshold [22, 23].

Considering that the stochastic process model can provide flexibility to describe failure generating mechanisms and operating environment characteristics, many articles used stochastic process approach to model degradation path [24-26], such as the Wiener process and Gamma process. In addition, most of the above papers assumed that the continuous degradation path was represented by a linear function of time $t$, but the nonlinearity exists extensively in practice [27]. It is worth pointing out that little research has been carried out to consider nonlinearity stochastic process and changing failure threshold for system experiencing DCFP and those have become a critical issue for many complex systems. In this paper, some new reliability evaluation models are conducted on the basis of DCFP. Firstly, some reliability models with hard failure and soft failure are established, where the soft failure process is governed by the random effect nonlinear Wiener process. On that basis, the DCFP models under the different shifting threshold are considered. $\mathrm{Nu}$ merical example indicates that the shocks and the shifting threshold have significant effects on system reliability.

The remainder of this paper is organized as follows. Failure process including degradation and random shock is described in Section 2. Some reliability models about hard failure and soft failure are established in Section 3. In Section 4, some new reliability models are obtained for system experiencing DCFP with different shifting thresholds. A numerical example is presented in Section 5, and the sensitivity analysis is also given. In Section 6, some main conclusions are obtained.

\section{Dependent Competing Failure Process}

Generally speaking, product may fail due to two different failure modes: soft failure and hard failure. These two failure processes are dependent because both of them are subject to the same shock. The product will fail when either of them exceeds its corresponding threshold value. In this section, the cumulative shock model is applied.

As shown in Figure 1(a), let $X(t)$ be the degradation volume of the continuous degradation until time $t$. Shock loads will cause additional abrupt damage, $Y_{i}(i=1,2,3$,
...,), which contribute to acceleration of the degradation process. When the overall degradation $X_{s}(t)$ is beyond critical strength level $H_{1}$, the soft failure occurs.

Considering that so many external shocks can affect the degradation performance of the product, it is a reasonable assumption that the product may become more vulnerable after multiple shocks. Therefore, in Figure 1(b), we suppose that the critical value of degradation failure decreases from $H_{1}$ to $H_{2}$ after the arrival of $m$ shocks and $m$ is the number of shocks' occurrences.

In addition, the same shock loads may also result in hard failure, as shown in Figure 2(a). Let $W_{i}$ denote the magnitude of $i$ th $(i=1,2,3, \ldots)$ shock, and hard failure occurs when cumulative shock loads magnitude exceeds threshold value $D_{1}$. Similarly, the hard threshold value $D_{1}$ can be affected by the random shock process. In Figure 2(b), the critical strength value decreases from $D_{1}$ to $D_{2}$ after the arrival of $m$ shocks, and $m$ is the number of shocks' occurrences.

\section{Reliability Modeling of Hard Failure and Soft Failure}

3.1. Reliability Modeling of Hard Failure due to Random Shock. During normal use stage, suppose that the product is affected by random shock, and random shocks reach according to the Poisson process with rate $\lambda$. Let $N(t)$ denote the total number of shocks in given time interval $(0, t]$. Then, the probability function of $N(t)$ can be obtained as

$$
P\{N(t)=i\}=\frac{(\lambda t)^{i}}{i !} e^{-\lambda t}, \quad i=0,1,2, \ldots .
$$

Let $W_{i}$ denote the magnitude of the $i$ th shock, and assume that $W_{i}$ is an independent and identically distributed random variable following a normal distribution as $W_{i} \sim N$ $\left(\mu_{w} \sigma_{w}^{2}\right)$ and $W_{0}=0$, and then we can obtain

$$
P\left(W_{i} \leq x\right)=\Phi\left(\frac{x-\mu_{w}}{\sigma_{w}}\right) .
$$

Let $S_{i}$ denote the total damage of the $i$ th shock magnitude $W_{i}(i=1,2,3, \ldots)$; then, we can obtain

$$
S_{i}=\sum_{j=1}^{i} W_{i}, \quad i \geq 1 .
$$

Via the property that the sum from independent normal distribution is still normal distribution, by using equations (2) and (3), the probability function of the total damage $S_{i}$ can be obtained as

$$
P\left(S_{i} \leq x\right)=\Phi\left(\frac{x-i \mu_{w}}{\sqrt{i} \sigma_{w}}\right) .
$$

Let $D(t)$ denote the total damage in given time interval $(0, t]$ and $N(t)$ denote the total number of shocks until time $t$. Then, we can obtain

$$
D(t)=\sum_{i=1}^{N(t)} W_{i}=S_{N(t)} .
$$




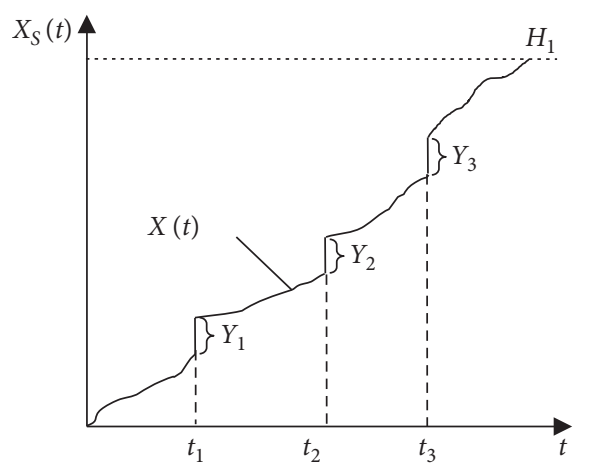

(a)

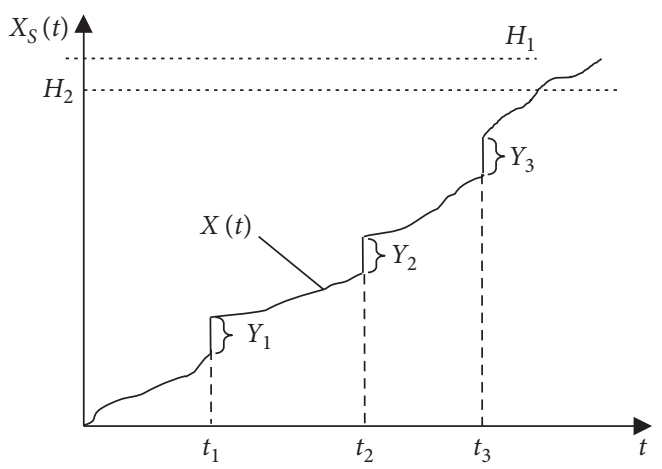

(b)

Figure 1: Soft failure process: (a) fixed threshold; (b) shifting threshold.

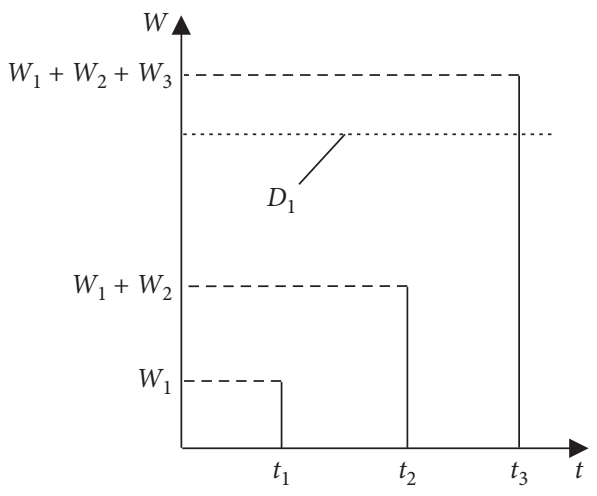

(a)

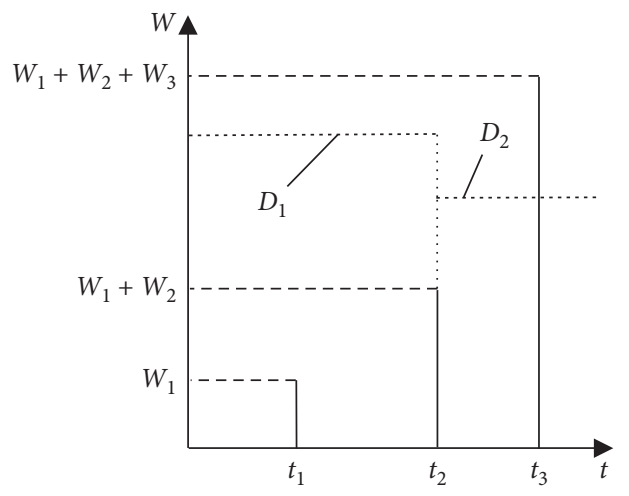

(b)

FIgURE 2: Hard failure process: (a) fixed threshold; (b) shifting threshold.

3.1.1. Case I: Reliability Analysis under a Fixed Hard Threshold Value. As shown in Figure 2(a), let $T$ be the hard failure time and $D_{1}$ be threshold value of the hard failure. By using the total probability formula and the compound Poisson process (5), the reliability of hard failure can be obtained as

$$
\begin{aligned}
R_{D}(t) & =P(T>t)=P\left(D(t)<D_{1}\right) \\
& =P\left(\sum_{j=0}^{N(t)} W_{j}<D_{1}\right)=\sum_{i=0}^{\infty} P\left(\sum_{j=0}^{N(t)} W_{j}<D_{1}, N(t)=i\right) \\
& =\sum_{i=0}^{\infty} P\left(\sum_{j=0}^{N(t)} W_{j}<D_{1} \mid N(t)=i\right) \cdot P(N(t)=i) \\
& =P(N(t)=0)+\sum_{i=1}^{\infty} P\left(\sum_{j=1}^{N(t)} W_{j}<D_{1} \mid N(t)=i\right) \cdot P(N(t)=i) \\
& =e^{-\lambda t}+\sum_{i=1}^{\infty} \Phi\left(\frac{\left(D_{1}-i \mu_{W}\right)}{\sqrt{i \sigma_{W}^{2}}}\right) \cdot \frac{e^{-\lambda t}}{i !}(\lambda t)^{i} .
\end{aligned}
$$


3.1.2. Case II: Reliability Analysis under a Shifting Hard Threshold Value. As shown in Figure 2(b), after $m$ shocks, the hard failure critical value decreases from $D_{1}$ to $D_{2}$.
Similarly, in such a shifting hard threshold situation, the reliability function of hard failure can be obtained as

$$
\begin{aligned}
R_{D}(t)= & P(T>t)=P\left(\sum_{j=1}^{m} W_{j}<D_{1}, \sum_{j=m+1}^{N(t)} W_{j}<D_{2}\right) \\
= & \sum_{i=0}^{m} P\left(\sum_{j=1}^{N(t)} W_{j}<D_{1}, N(t)=i\right)+\sum_{i=m+1}^{\infty} P\left(\sum_{j=1}^{N(t)} W_{j}<D_{2}, N(t)=i\right) \\
= & \left.P(N(t)=0)+\sum_{i=1}^{m} P\left(\sum_{j=1}^{i} W_{j}<D_{1} \mid N(t)=i\right)\right) \cdot P(N(t)=i) \\
& +\sum_{i=m+1}^{\infty} P\left(\sum_{j=1}^{i} W_{j}<D_{2} \mid N(t)=i\right) \cdot P(N(t)=i) \\
= & e^{-\lambda t}+\sum_{i=1}^{m} \Phi\left(\frac{\left(D_{1}-i \mu_{W}\right)}{\sqrt{i \sigma_{W}^{2}}}\right) \cdot \frac{e^{-\lambda t}}{i !}(\lambda t)^{i}+\sum_{i=m+1}^{\infty} \Phi\left(\frac{\left(D_{2}-i \mu_{W}\right)}{\sqrt{i \sigma_{W}^{2}}}\right) \cdot \frac{e^{-\lambda t}}{i !}(\lambda t)^{i},
\end{aligned}
$$

where $m$ is the number of shocks' occurrences when failure threshold decreases.

\subsection{Reliability Modeling of Soft Failure due to Degradation and} Shock. Considering that the nonlinearity exists extensively in practice and the linear model cannot trace the dynamics, a nonlinearity stochastic process is used to describe the degradation path. A well-adopted form for the nonlinearity Wiener process $\{X(t), t \geq 0\}$ can be expressed as

$$
X(t)=a \Lambda(t, b)+\sigma B(\Lambda(t, b)),
$$

where $a$ is the drift degradation rate, $\sigma$ is the diffusion coefficient, $\Lambda(t)$ is the transformed time scale, and $B(\cdot)$ is the standard Brownian motion representing a time correlated structure.

Considering that product possibly experiences different sources of variations during its operation, for a degradation model to be realistic, it is more appropriate to incorporate unit to unit variability in the degradation process. To capture the unit to unit differences, the random effect model is used in this paper. The nonlinearity degradation model with random effect can be expressed as

$$
\left\{\begin{array}{l}
X(t)=a \Lambda(t, b)+\sigma B(\Lambda(t, b)), \\
a \sim N\left(\mu_{a}, \sigma_{a}^{2}\right) .
\end{array}\right.
$$

For simplicity, we assume that random variables $a$ and $B(t)$ are independent. From the properties of the Wiener process, we can obtain that the nonlinearity degradation performance $X(t)$ is normally distributed as

$$
X(t) \sim N\left(\mu_{a} \Lambda(t, b), \sigma_{a}^{2}(\Lambda(t, b))^{2}+\sigma^{2} \Lambda(t, b)\right) .
$$

3.2.1. Case III: Reliability Analysis under Fixed Degradation Threshold Value. Suppose that the random shock can cause an abrupt damage. The abrupt damages are measured by the shock damage sizes as $\left\{Y_{1}, Y_{2}, \ldots,\right\}$. Let $S(t)$ be the cumulative damage size due to random shocks until time $t$; then, we can obtain

$$
S(t)= \begin{cases}\sum_{j=1}^{N(t)} Y_{j}, & \text { if } N(t)>0 \\ 0, & \text { if } N(t)=0 .\end{cases}
$$

As shown in Figure 1(a), the total damage of system $X_{S}(t)$ is the sum of the cumulative effect of continuous degradation damage and abrupt degradation damages due to shocks until time $t$. The soft failure occurs when the overall degradation is beyond the threshold value $H_{1}$. From Figure 1(a), we can obtain

$$
X_{S}(t)=X(t)+S(t)
$$

In this article, we assume that the magnitude of gradual degradation $X(t)$ follows normal distribution as $N\left(\mu(t), \sigma^{2}\right.$ $(t))$. And the shock damage sizes $Y_{i}$ are independently distributed random variables taking the form as $Y_{i} \sim N$ $\left(\mu_{Y} \sigma_{Y}^{2}\right)$. Considering the independence of the two random variables, $X_{s}(t)$ follows the Gaussian distribution as

$$
\begin{aligned}
X_{s}(t) \sim & N\left(\mu_{a} \Lambda(t, b)+E[N(t)] \mu_{Y}, \sigma_{a}^{2}(\Lambda(t, b))^{2}+\sigma^{2} \Lambda(t, b)\right. \\
& \left.+E[N(t)] \sigma_{Y}^{2}\right) .
\end{aligned}
$$

In most engineering problems, the mean and standard deviation of a degradation process increase in terms of time. The Gaussian process with increasing mean and standard 
deviation could be used to describe these engineering problems. Furthermore, when $X_{s}(t)$ is a Gaussian process with increasing mean and standard deviation, $R_{s}(t)=P\left(X_{s}(t)<H_{1}\right)$ is a monotone decreasing function with respect to time.
As shown in Figure 1(a), let $T$ be the soft failure time and $H_{1}$ be the corresponding threshold value. Then, considering the monotone increasing features, the reliability function of the soft failure process can be obtained as

$$
\begin{aligned}
R_{s}(t)= & P(T>t)=P\left(X_{s}(t)<H_{1}\right) \\
= & P\left(X(t)+S(t)<H_{1}\right) \\
= & P\left(X(t)<H_{1} \mid N(t)=0\right) P(N(t)=0) \\
& +\sum_{i=1}^{\infty} P\left(X(t)+\sum_{j=1}^{N(t)} Y_{j}<H_{1} \mid N(t)=i\right) \cdot P(N(t)=i) \\
= & \Phi\left(\frac{H_{1}-\mu_{a} \Lambda(t, b)}{\sqrt{\sigma_{a}^{2}(\Lambda(t, b))^{2}+\sigma^{2} \Lambda(t, b)}}\right) \cdot e^{-\lambda t} \\
& +\sum_{i=1}^{\infty} \Phi\left(\frac{H_{1}-\left(i \mu_{Y}+\mu_{a} \Lambda(t, b)\right)}{\sqrt{\sigma_{a}^{2}(\Lambda(t, b))^{2}+\sigma^{2} \Lambda(t, b)+i \sigma_{Y}^{2}}}\right) \cdot \frac{e^{-\lambda t}}{i !}(\lambda t)^{i} .
\end{aligned}
$$

3.2.2. Case IV: Reliability Analysis under a Shifting Degradation Threshold Value. As shown in Figure 1(b), after $m$ shocks, the soft failure critical value decreases from $\mathrm{H}_{1}$ to $\mathrm{H}_{2}$.
In such a shifting threshold situation, the reliability function can be obtained as

$$
\begin{aligned}
R_{s}(t)= & P\left(X_{s}(t)<H_{1} \mid N(t)=0\right) P(N(t)=0) \\
& +\sum_{i=1}^{m} P\left(X_{s}(t)<H_{1} \mid N(t)=i\right) P(N(t)=i) \\
& +\sum_{i=m+1}^{\infty} P\left(X_{s}(t)<H_{2} \mid(t)=i\right) P(N(t)=i) \\
= & P\left(X(t)<H_{1}\right) \cdot P(N(t)=0) \\
& +\sum_{i=1}^{m} P\left(X(t)+\sum_{j=1}^{i} Y_{j}<H_{1}\right) \cdot P(N(t)=i) \\
& +\sum_{i=m+1}^{\infty} P\left(X(t)+\sum_{j=1}^{i} Y_{j}<H_{2}\right) \cdot P(N(t)=i) \\
= & \left(\frac{H_{1}-\mu \Lambda(t)}{\sqrt{\sigma_{a}^{2}(\Lambda(t, b))^{2}+\sigma^{2} \Lambda(t, b)}}\right) e^{-\lambda t}+\sum_{i=1}^{m} \Phi\left(\frac{H_{1}-\left(i \mu_{Y}+\mu \Lambda(t)\right)}{\sqrt{\sigma_{a}^{2}(\Lambda(t, b))^{2}+\sigma^{2} \Lambda(t, b)+i \sigma_{Y}^{2}}}\right) \cdot \frac{e^{-\lambda t}}{i !}(\lambda t)^{i} \\
& +\sum_{i=m+1}^{\infty} \Phi\left(\frac{H_{2}-\left(i \mu_{Y}+\mu \Lambda(t)\right)}{\sqrt{\sigma_{a}^{2}(\Lambda(t, b))^{2}+\sigma^{2} \Lambda(t, b)+i \sigma_{Y}^{2}}}\right) \cdot \frac{e^{-\lambda t}}{i !}(\lambda t)^{i} .
\end{aligned}
$$




\section{Reliability Modeling and Analysis for DCFP}

From the above analysis, the reliability functions under hard failure and soft failure are established, respectively. In the following research, considering that the system subjects to both degradation and random shocks, some new dependent competing reliability modeling and assessment models are established.

4.1. Reliability Analysis due to a Fixed Threshold Value. As shown in Figures 1(a) and 2(a), we can find that the system includes two types of dependent competing failure processes. Thus, the DCFP system reliability at time $t$ is the probability that the total degradation is less than the soft threshold level $\left(X_{s}(t)<H_{1}\right)$ and the cumulative magnitude is less than the hard threshold value $\left(\sum_{k=1}^{N(t)} W_{k}<D_{1}\right)$. That is to say, the system reliability function can be obtained as

$$
\begin{aligned}
& R(t)=P\left(X_{s}(t)<H_{1}, \sum_{k=1}^{N(t)} W_{k}<D_{1}\right) \\
& =P\left(X(t)+\sum_{j=1}^{N(t)} Y_{j}<H_{1}, \sum_{k=1}^{N(t)} W_{k}<D_{1}\right) \\
& =P\left(X(t)<H_{1} \mid N(t)=0\right) P(N(t)=0) \\
& +\sum_{i=1}^{\infty} P\left(X(t)+\sum_{j=1}^{i} Y_{j}<H_{1}, \sum_{k=1}^{i} W_{k}<D_{1} \mid N(t)=i\right) \\
& \text { - } P(N(t)=i) \\
& =P\left(X(t)<H_{1} \mid N(t)=0\right) P(N(t)=0) \\
& +\sum_{i=1}^{\infty} P\left(\sum_{k=1}^{i} W_{k}<D_{1}\right) P\left(X(t)+\sum_{j=1}^{i} Y_{j}<H_{1}\right) \\
& \text { - } P(N(t)=i) \\
& =\Phi\left(\frac{H_{1}-\mu_{a} \Lambda(t, b)}{\sqrt{\sigma_{a}^{2}(\Lambda(t, b))^{2}+\sigma^{2} \Lambda(t, b)}}\right) e^{-\lambda t} \\
& +\sum_{i=1}^{\infty} \Phi\left(\frac{D_{1}-i \mu_{W}}{\sqrt{i} \sigma_{W}}\right) \Phi\left(\frac{H_{1}-\left(i \mu_{Y}+\mu_{a} \Lambda(t, b)\right)}{\sqrt{\sigma_{a}^{2}\left(\Lambda(t, b)^{2}+\sigma^{2} \Lambda(t, b)\right)+i \sigma_{Y}^{2}}}\right) \\
& \cdot \frac{e^{-\lambda t}}{i !}(\lambda t)^{i}
\end{aligned}
$$

4.2. Reliability Analysis due to a Shifting Hard Threshold Value. As shown in Figure 2(b), after the shocks happened $m$ times, the hard failure threshold decreases from $D_{1}$ to $D_{2}$. In such a shifting hard threshold situation, we can get the DCFP system reliability at time $t$ as

$$
\begin{aligned}
& R(t)=P\left(X_{s}(t)<H_{1}, N(t)=0\right) \\
& +\sum_{i=1}^{m} P\left(X_{s}(t)<H_{1}, \sum_{k=1}^{N(t)} W_{k}<D_{1}, N(t)=i\right) \\
& +\sum_{i=m+1}^{\infty} P\left(X_{s}(t)<H_{1}, \sum_{k=1}^{N(t)} W_{k}<D_{2}, N(t)=i\right) \\
& =P\left(X_{s}(t)<H_{1} \mid N(t)=0\right) P(N(t)=0) \\
& +\sum_{i=1}^{m} P\left(X_{s}(t)<H_{1}, \sum_{k=1}^{N(t)} W_{k}<D_{1} \mid N(t)=i\right) P(N(t)=i) \\
& +\sum_{i=m+1}^{\infty} P\left(X_{s}(t)<H_{1}, \sum_{k=1}^{N(t)} W_{k}<D_{2} \mid N(t)=i\right) P(N(t)=i) \\
& =P\left(X(t)<H_{1}\right) \cdot P(N(t)=0) \\
& +\sum_{i=1}^{m} P\left(\sum_{k=1}^{i} W_{k}<D_{1}\right) P\left(X(t)+\sum_{j=1}^{i} Y_{j}<H_{1}\right) \cdot P(N(t)=i) \\
& +\sum_{i=m+1}^{\infty} P\left(\sum_{k=1}^{i} W_{k}<D_{2}\right) P\left(X(t)+\sum_{j=1}^{i} Y_{j}<H_{1}\right) \\
& \cdot P(N(t)=i) \\
& =\Phi\left(\frac{H_{1}-\mu_{a} \Lambda(t)}{\sqrt{\sigma_{a}^{2}(\Lambda(t, b))^{2}+\sigma^{2} \Lambda(t, b)}}\right) e^{-\lambda t} \\
& +\sum_{i=1}^{m} \Phi\left(\frac{D_{1}-i \mu_{W}}{\sqrt{i} \sigma_{W}}\right) \Phi\left(\frac{H_{1}-\left(i \mu_{Y}+\mu_{a} \Lambda(t)\right)}{\sqrt{\sigma_{a}^{2}(\Lambda(t, b))^{2}+\sigma^{2} \Lambda(t, b)+i \sigma_{Y}^{2}}}\right) \\
& \cdot \frac{e^{-\lambda t}}{i !}(\lambda t)^{i} \\
& +\sum_{i=m+1}^{\infty} \Phi\left(\frac{D_{2}-i \mu_{W}}{\sqrt{i} \sigma_{W}}\right) \Phi\left(\frac{H_{1}-\left(i \mu_{Y}+\mu_{a} \Lambda(t)\right)}{\sqrt{\sigma_{a}^{2}(\Lambda(t, b))^{2}+\sigma^{2} \Lambda(t, b)+i \sigma_{Y}^{2}}}\right) \\
& \cdot \frac{e^{-\lambda t}}{i !}(\lambda t)^{i}
\end{aligned}
$$

4.3. Reliability Analysis due to a Shifting Soft Threshold Value. Similar to Section 4.2, in Figure 1(b), after the shocks happened $m$ times, the soft failure threshold decreases from $H_{1}$ to $H_{2}$. In such a shifting soft failure threshold situation, we can get DCFP system reliability at time $t$ as 


$$
\begin{aligned}
& R(t)=P\left(X_{s}(t)<H_{1}, N(t)=0\right) \\
& +\sum_{i=1}^{m} P\left(\sum_{k=1}^{N(t)} W_{k}<D_{1}, X_{s}(t)<H_{1}, N(t)=i\right) \\
& +\sum_{i=m+1}^{\infty} P\left(\sum_{k=1}^{N(t)} W_{k}<D_{1}, X_{s}(t)<H_{2}, N(t)=i\right) \\
& =P\left(X_{s}(t)<H_{1} \mid N(t)=0\right) P(N(t)=0)+\sum_{i=1}^{m} P\left(\sum_{k=1}^{N(t)} W_{k}<D, X_{s}(t)<H_{1} \mid N(t)=i\right) P(N(t)=i) \\
& +\sum_{i=m+1}^{\infty} P\left(\sum_{k=1}^{N(t)} W_{k}<D, X_{s}(t)<H_{2} \mid N(t)=i\right) P(N(t)=i) \\
& =P\left(X(t)<H_{1}\right) \cdot P(N(t)=0) \\
& +\sum_{i=1}^{m} P\left(\sum_{k=1}^{i} W_{k}<D_{1}\right) P\left(X(t)+\sum_{j=1}^{i} Y_{j}<H_{1}\right) \cdot P(N(t)=i) \\
& +\sum_{i=m+1}^{\infty} P\left(\sum_{k=1}^{i} W_{k}<D_{1}\right) P\left(X(t)+\sum_{j=1}^{i} Y_{j}<H_{2}\right) \cdot P(N(t)=i) \\
& =\Phi\left(\frac{H_{1}-\mu_{a} \Lambda(t)}{\sqrt{\sigma_{a}^{2}(\Lambda t, b)^{2}+\sigma^{2} \Lambda(t, b)}}\right) e^{-\lambda t}+\sum_{i=1}^{m} \Phi\left(\frac{D_{1}-i \mu_{W}}{\sqrt{i} \sigma_{W}}\right) \Phi\left(\frac{H_{1}-\left(i \mu_{Y}+\mu_{a} \Lambda(t)\right)}{\sqrt{\sigma_{a}^{2}(\Lambda(t, b))^{2}+\sigma^{2} \Lambda(t, b)+i \sigma_{Y}^{2}}}\right) \cdot \frac{e^{-\lambda t}}{i !}(\lambda t)^{i} \\
& +\sum_{i=m+1}^{\infty} \Phi\left(\frac{D_{1}-i \mu_{W}}{\sqrt{i} \sigma_{W}}\right) \Phi\left(\frac{H_{2}-\left(i \mu_{Y}+\mu_{a} \Lambda(t)\right)}{\sqrt{\sigma_{a}^{2}(\Lambda(t, b))^{2}+\sigma^{2} \Lambda(t, b)+i \sigma_{Y}^{2}}}\right) \cdot \frac{e^{-\lambda t}}{i !}(\lambda t)^{i}
\end{aligned}
$$

\section{Numerical Example: A Fatigue Crack Growth Data Application}

In this section, a case study is given to illustrate the proposed models and methods. This example is based on the fatigue crack growth data of an alloy in paper from Lu and Meeker [28]. The datasets were collected from 13 test samples, and all samples have the same initial crack length of 0.90 inches. The loading cycles of the datasets are considered to be the loading time. Figure 3 shows part of the data, and time unit is in $10^{4}$ cycles.

Similarly to [13], we suppose sizes of random shock loads, $W_{i}(i=1,2,3, \ldots$,$) , are assumed to follow normal distribution,$ $W_{i} \sim N(2,0.5)$, which are measured in units of component life. The shock damage sizes $Y_{i} \sim N(0.02,0.01)$, the degradation failure threshold value $H=2.0$ inches, catastrophic failure threshold value $D_{1}=35$ units, and the parameter $\lambda=0.5$. In addition, we assume that the number $m=3$, the lower level of degradation failure threshold value $H_{2}=1.8 \mathrm{~mm}$, and the lower level of catastrophic failure threshold value $D_{2}=25$ units.

5.1. Estimation of Unknown Parameters. Firstly, we use the random effect nonlinear Wiener process model to fit the fatigue crack growth data. According to the suggestion of Pan et al. [29], let $\Lambda(t)=\Lambda(t, b)=t^{b}$ be a reasonable choice for the degradation modeling about the fatigue crack growth data.

Here, we discuss the estimation of parameters required to implement the reliability function in equation (9). The unknown parameters are

$$
\theta=\left(\mu_{\alpha}, \sigma_{\alpha}, \sigma, b\right) .
$$

By using MCMC method and WinBUGS [30], the parameters posterior mean, standard error, Monte Carlo error, and 95\% HPD interval are obtained in Table 1.

5.2. Reliability Analysis Subject to Only Hard Failure or Soft Failure. According to equations (6), (7), (14), and (15), under the fixed and shifting failure threshold, the reliability curves are plotted in Figures 4 and 5, respectively. From Figures 4 and 5, we can obtain that the shifting threshold have significant effect on the failure process, both hard failure and soft failure.

5.3. System Reliability Analysis Subject to DCFP. According to equations (16)-(18), the reliability curve of different cases in Section 4 are plotted in Figure 6, 


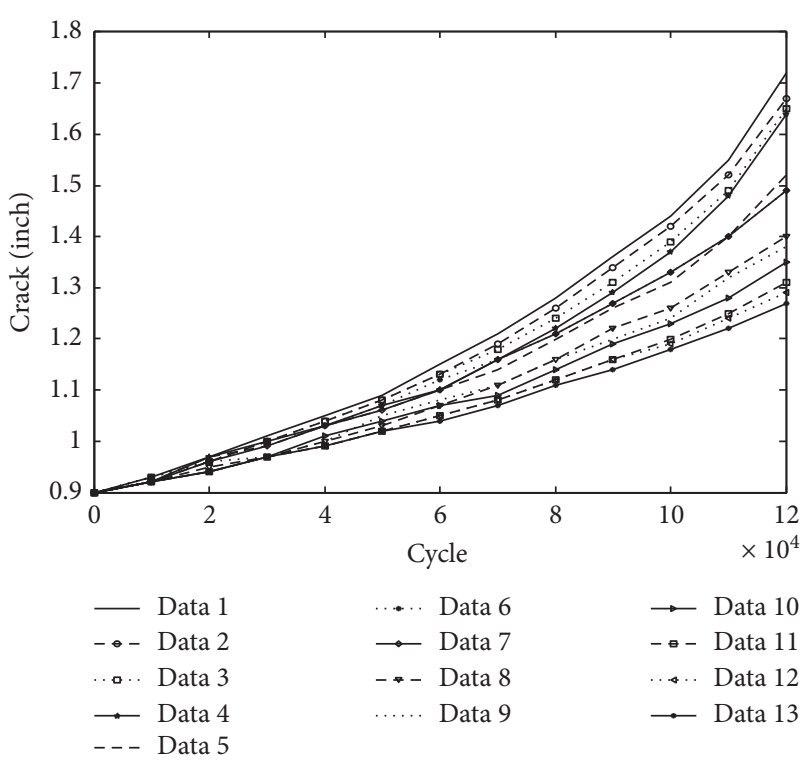

FIgURE 3: Fatigue crack growth paths of samples.

TABLE 1: Estimation results of the unknown parameters.

\begin{tabular}{lcccc}
\hline Parameter & Mean & $\begin{array}{c}\text { Standard } \\
\text { error }\end{array}$ & MC error & 95\% HPD interval \\
\hline$\mu_{a}$ & 0.01657 & 0.03197 & $5.579 E-4$ & $(0.007272,0.02521)$ \\
$\sigma_{a}$ & 0.01475 & 0.007836 & $9.793 E-5$ & $(0.009824,0.02282)$ \\
$\sigma$ & 0.01010 & 0.01359 & $2.847 E-4$ & $(0.008515,0.01148)$ \\
$b$ & 1.44100 & 0.05458 & 0.002569 & $(1.443,1.508)$ \\
\hline
\end{tabular}

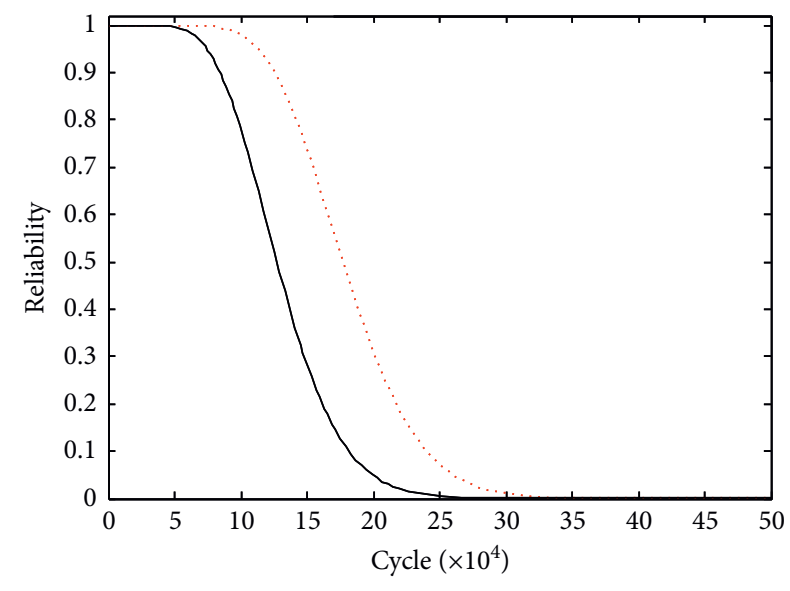

... Reliability of the fixed hard threshold

— Reliability of the shifting hard threshold

FIgURE 4: Reliability curves subject to hard failure.

respectively. From Figure 6, we can obtain the following conclusions:

(a) Compared with the degradation process without shocks, the reliability of system with shocks is

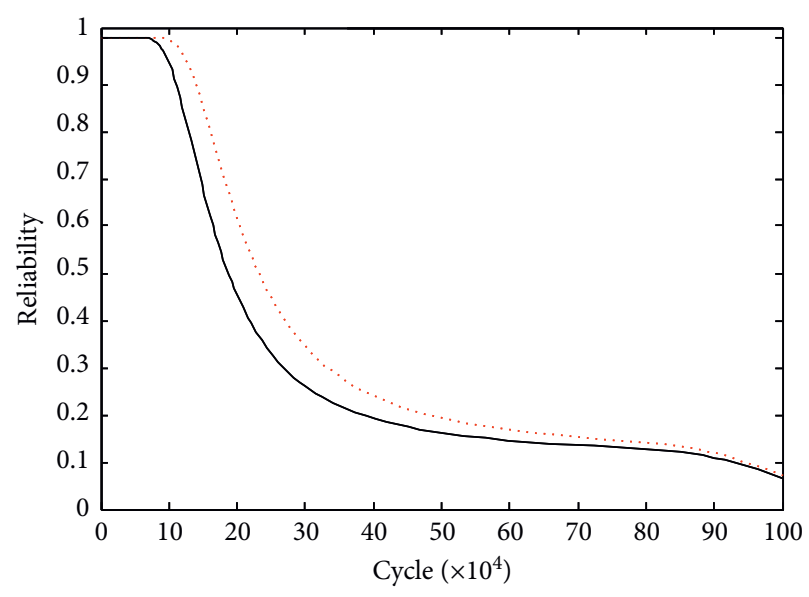

Reliability of the fixed soft threshold

_ Reliability of the shifting soft threshold

FIgURE 5: Reliability curves subject to soft failure.

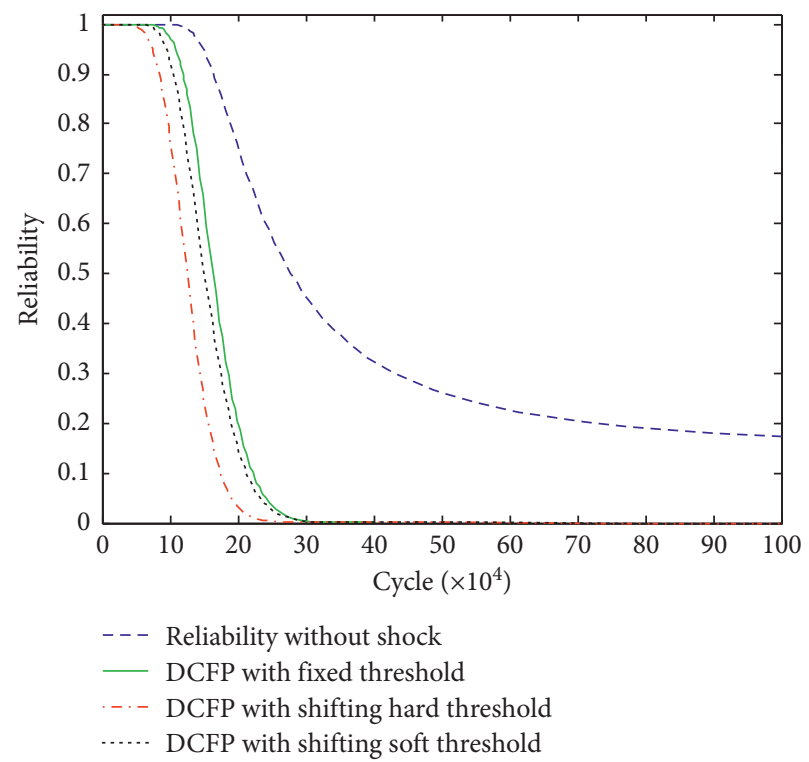

FIgURE 6: Reliability curves of different models.

significantly lower. Conclusion indicates that the shocks have significant effect on the reliability of system.

(b) When system sustains a series of shocks, it is easier to fail. Conclusion indicates that the shock can decrease the failure threshold, both the soft failure and hard failure threshold. Thus, when the shifting thresholds are considered, the reliability of system is also significantly lower than the fixed thresholds.

(c) For different cases, we can find that the reliability almost keeps 1 when $t<10^{5}$ cycles because the effect of random shocks is not significant during that time, and the gradual degradation amount is not large enough to cause any failure. In the next time period, with the gradual degradation and random shocks, 


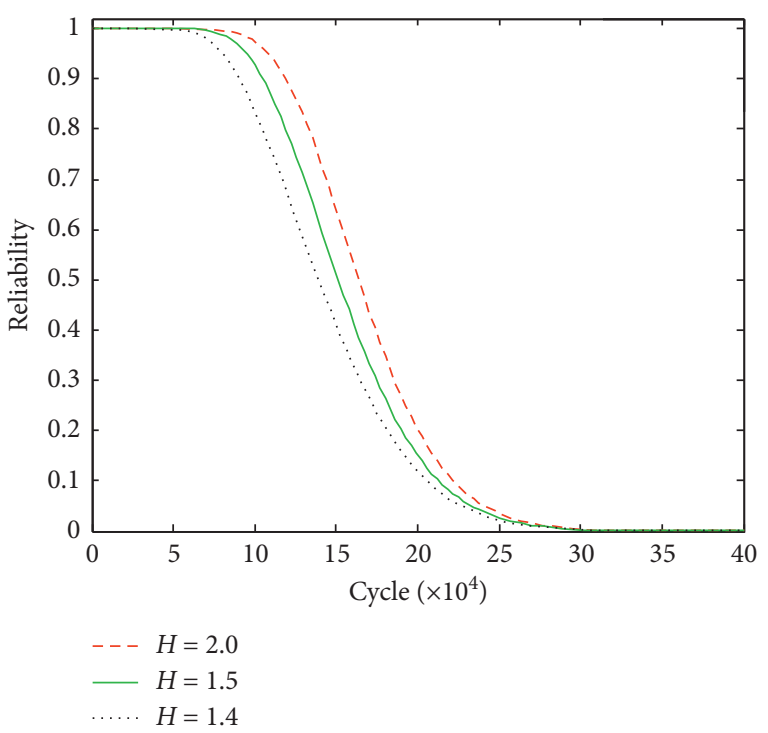

Figure 7: Sensitivity analysis of $R(t)$ and on $H$.

reliability is decreasing quickly; the random shocks become a significant factor for the system reliability. Eventually, when $t>2.5 \times 10^{5}$ cycles, reliability drops to a quite low level.

Reliability curve based on degradation without shocks is also provided in Figure 6. According to the results, it can be concluded that random shocks will accelerate the failure process which validates the effectiveness of models constructed in Sections 3 and 4. Moreover, when hard failure threshold value decreases, failure will occur sooner.

5.4. Sensitivity Analysis of Different Parameters. This section only discusses the sensitivity analysis of the fixed threshold case, and different shifting threshold cases can be analyzed similarly.

Sensitivity analysis under the threshold $H_{1}$ of soft failure, the threshold $D_{1}$ of hard failure, and the random shocks rate $\lambda$ are investigated with regard to reliability distribution, and the corresponding curve are plotted in Figures 7-9, respectively.

From Figures 7 and 8, we can find that both soft failure threshold and hard failure threshold have significant effect on the reliability performance. Reliability curve shifts to right as the threshold value increases, which implies a better reliability performance with a larger threshold value. However, the changing thresholds by man-modified can improve reliability, but that does not mean system becomes more reliable. In engineering practice, many factors should be considered comprehensively in order to scientifically determine the reliability threshold, such as the system characteristics factor, operating conditions factor, and working environment factor.

In addition, by comparing Figures 7 and 8 , it can be found that the influence of soft failure threshold on system reliability exists in the whole life cycle; however, the hard failure threshold has different influences on system

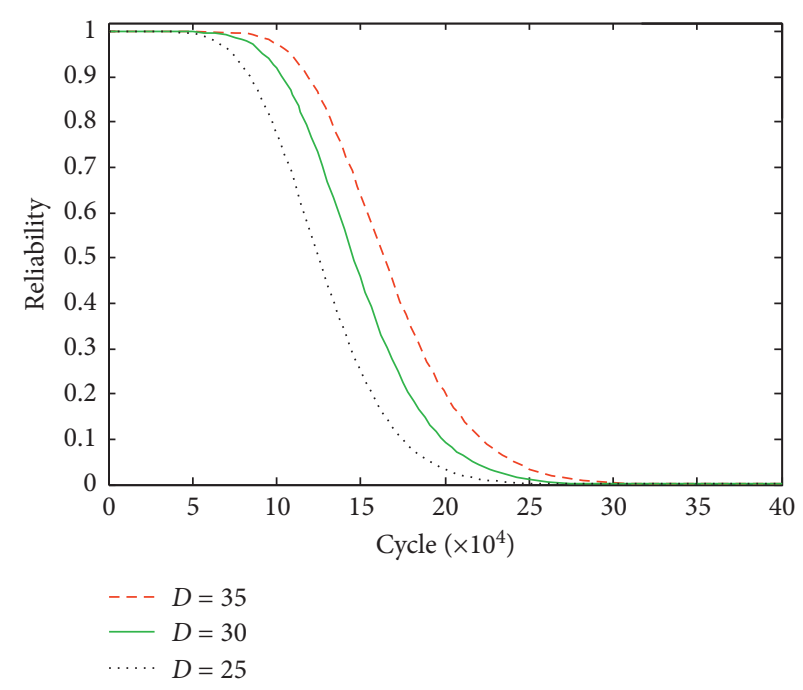

FIgURE 8: Sensitivity analysis of $R(t)$ and on $D$.

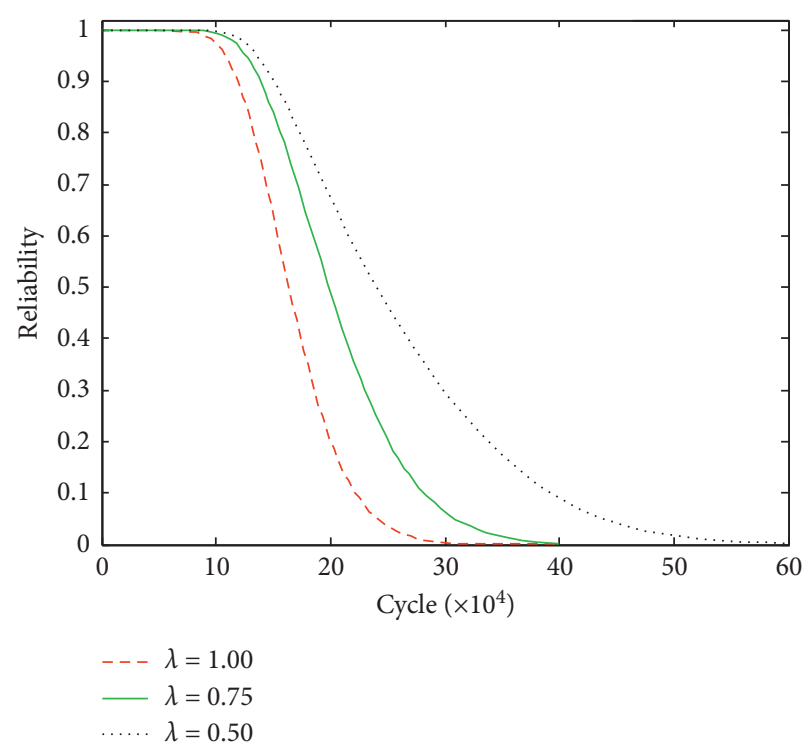

FIgure 9: Sensitivity analysis of $R(t)$ and on $\lambda$.

reliability in different time periods, and the closer it is to the end of the life cycle, the greater the influence will be. This is because the number of initial shocks is small, and the cumulative effect of shocks is not obvious. After a period of time, the cumulative impact effect has been very significant, and if hard failure threshold drops, the impact on system reliability will become more obvious.

Figure 9 shows that the shock arrival rate, $\lambda$, has a significant effect with system reliability. Obviously, comparing with the effect of the failure threshold, the reliability is more sensitive for the change of impact arrival rate $\lambda$. It is reasonable to get such results because intensively frequent random shocks, larger sizes of shock loads, and shock damages will resultantly make a component more vulnerable. Therefore, when the number of shocks is large enough, 
the reliability of the system will drop to a very low level; the higher the impact arrival rate, the earlier the system enters the unreliable state.

Therefore, by setting higher failure threshold $H_{1}, H_{2}, D_{1}$, and $D_{2}$, the system seems to be more reliable. However, it is very difficult to make such assumption in reality, since reliability threshold depends on many conditions, including manufacturing processes, importance, work environment, and maintaining measures.

\section{Conclusions}

In this paper, some new reliability evaluation models are established by considering dependent competing failure process due to soft failure and hard failure under different shifting thresholds. A real numerical example about fatigue crack growth dataset is carried out to demonstrate the proposed procedure. Compared with the degradation process without shocks, models established in this paper indicate that random shocks have significant effect on the failure process. When considering the shifting-threshold situation, reliability decreases even faster. From the sensitivity analysis, the shifting threshold and the shock arrival rate have a significant effect with the system reliability.

The proposed models in this paper only consider one degradation path. In real situation, components may have multiple degradation measures, and this will be the focus of future research. In addition, this paper only considers the cumulative shock model and wiener process model; in the future, some other works can be carried out, such as extreme shock model, $\delta$-shock model, Gamma process model, and inverse Gaussian process model.

\section{Data Availability}

The data used to support the findings of this study are available from the corresponding author upon request.

\section{Conflicts of Interest}

The authors declare that there are no conflicts of interest regarding the publication of this article.

\section{Authors' Contributions}

The authors contributed equally to the presented mathematical framework and the writing of the paper. All authors have read and approved the final manuscript.

\section{Acknowledgments}

This work was supported by the Humanity and Social Science Foundation of Ministry of Education of China (no. 19YJAZH039), Key Project of Hubei Provincial Education Department (no.D20172701), Technology Creative Project of Excellent Middle \& Young Team of Hubei Province (no. T201920), National Bureau of Statistics of China (no.2017LY73), and Humanity and Social Science Foundation of Ministry of Education of China (no. 20YJAZH035).

\section{References}

[1] W. Li and H. Pham, "Reliability modeling of multi-state degraded systems with multi-competing failures and random shocks," IEEE Transactions on Reliability, vol. 54, no. 2, pp. 297-303, 2005.

[2] A. Lehmann, "Joint modeling of degradation and failure time data," Journal of Statistical Planning and Inference, vol. 139, no. 5, pp. 1693-1706, 2009.

[3] G.-A. Klutke and Y. Yang, "The availability of inspected systems subject to shocks and graceful degradation," IEEE Transactions on Reliability, vol. 51, no. 3, pp. 371-374, 2002.

[4] Y. Zhu, E. A. Elsayed, H. Liao, and L. Y. Chan, "Availability optimization of systems subject to competing risk," European Journal of Operational Research, vol. 202, no. 3, pp. 781-788, 2010.

[5] E. Deloux, B. Castanier, and C. Bérenguer, "Predictive maintenance policy for a gradually deteriorating system subject to stress," Reliability Engineering \& System Safety, vol. 94, no. 2, pp. 418-431, 2009.

[6] M. Kijima and T. Nakagawa, "A cumulative damage shock model with imperfect preventive maintenance," Naval Research Logistics, vol. 38, no. 2, pp. 145-156, 1991.

[7] Y. Wang and H. Pham, "Imperfect preventive maintenance policies for two-process cumulative damage model of degradation and random shocks," International Journal of System Assurance Engineering and Management, vol. 2, no. 1, pp. 66-77, 2011.

[8] J. Chen and Z. Li, "An extended extreme shock maintenance model for a deteriorating system," Reliability Engineering \& System Safety, vol. 93, no. 8, pp. 1123-1129, 2008.

[9] G. Allan, "Mixed shock models," Bernoulli, vol. 7, no. 3, pp. 541-555, 2001.

[10] J.-M. Bai, Z.-H. Li, and X.-B. Kong, "Generalized shock models based on a cluster point process," IEEE Transactions on Reliability, vol. 55, no. 3, pp. 542-550, 2006.

[11] Y. Lam, "A geometric process delta-shock maintenance model," IEEE Transactions on Reliability, vol. 58, no. 2, pp. 389-396, 2009.

[12] H. Peng, Q. Feng, and D. W. Coit, "Reliability and maintenance modeling for systems subject to multiple dependent competing failure processes," IIE Transactions, vol. 43, no. 1, pp. 12-22, 2010.

[13] Z. Wang, H.-Z. Huang, Y. Li, and N.-C. Xiao, "An approach to reliability assessment under degradation and shock process," IEEE Transactions on Reliability, vol. 60, no. 4, pp. 852-863, 2011.

[14] Q. Qiu and L. Cui, "Reliability evaluation based on a dependent two-stage failure process with competing failures," Applied Mathematical Modelling, vol. 64, pp. 699-712, 2018.

[15] S. Song, D. W. Coit, Q. Feng, and H. Peng, "Reliability analysis for multi-component systems subject to multiple dependent competing failure processes," IEEE Transactions on Reliability, vol. 63, no. 1, pp. 331-345, 2014.

[16] K. Rafiee, Q. Feng, and D. W. Coit, "Reliability modeling for dependent competing failure processes with changing degradation rate," IIE Transactions, vol. 46, no. 5, pp. 483-496, 2014.

[17] Z. An and D. Sun, "Reliability modeling for systems subject to multiple dependent competing failure processes with shock loads above a certain level," Reliability Engineering \& System Safety, vol. 157, pp. 129-138, 2017.

[18] S. Hao and J. Yang, "Reliability analysis for dependent competing failure processes with changing degradation rate 
and hard failure threshold levels," Computers \& Industrial Engineering, vol. 118, pp. 340-351, 2018.

[19] M. Fan, Z. Zeng, E. Zio, R. Kang, and Y. Chen, "A sequential bayesian approach for remaining useful life prediction of dependent competing failure processes," IEEE Transactions on Reliability, vol. 68, no. 1, pp. 317-329, 2018.

[20] D. Kong, C. Qin, Y. He, and L. Cui, "Sensor-based calibrations to improve reliability of systems subject to multiple dependent competing failure processes," Reliability Engineering \& System Safety, vol. 160, pp. 101-113, 2017.

[21] H. D. Gao, L. R. Cui, and Q. G. Qiu, "Reliability modeling for degradation-shock dependence systems with multiple species of shocks," Reliability Engineering and System Safety, vol. 185, pp. 133-143, 2019.

[22] L. Jiang, Q. Feng, and D. W. Coit, "Reliability and maintenance modeling for dependent competing failure processes with shifting failure thresholds," IEEE Transactions on Reliability, vol. 61, no. 4, pp. 932-948, 2012.

[23] L. Jiang, Q. Feng, and D. W. Coit, "Reliability analysis for dependent failure processes and dependent failure threshold," in Proceedings of the 2011 International Conference on Quality, Reliability, Risk, Maintenance, and Safety Engineering, pp. 30-34, Xi'an, China, June 2011.

[24] Q. Dong, L. Cui, and S. Si, "Reliability and availability analysis of stochastic degradation systems based on bivariate wiener processes," Applied Mathematical Modelling, vol. 79, pp. 414-433, 2020.

[25] D. Kong and L. Cui, "Bayesian inference of multi-stage reliability for degradation systems with calibrations," Proceedings of the Institution of Mechanical Engineers, Part O: Journal of Risk and Reliability, vol. 230, no. 1, pp. 18-33, 2016.

[26] Q. A. Qiu and L. R. Cui, "Gamma process based optimal mission abort policy," Reliability Engineering and System Safety, vol. 190, Article ID 106496, 2019.

[27] X.-S. Si, W. Wang, C.-H. Hu, D.-H. Zhou, and M. G. Pecht, "Remaining useful life estimation based on a nonlinear diffusion degradation process," IEEE Transactions on Reliability, vol. 61, no. 1, pp. 50-67, 2012.

[28] C. J. Lu and W. O. Meeker, "Using degradation measures to estimate a time-to-failure distribution," Technometrics, vol. 35, no. 2, pp. 161-174, 1993.

[29] Z. Pan, N. Balakrishnan, Q. Sun, and J. Zhou, "Bivariate degradation analysis of products based on wiener processes and copulas," Journal of Statistical Computation and Simulation, vol. 83, no. 7, pp. 1316-1329, 2013.

[30] I. Ntzoufras, Bayesian Modeling Using WinBUGS, John Wiley \& Sons, New York City, NY, USA, 2009. 